\title{
miRNA and genes of the MYB plant family involved in the response to stress
}

Ivashchenko A.T.*, Rakhmetullina A.K., Pyrkova A.U. Al-Farabi Kazakh National University, Almaty, Kazakhstan

*e-mail:a.iavashchenko@gmail.com

MYB transcription factors (TFs) family play a great role in the development, metabolism and plant responses to biotic and abiotic stress. The free energy of miRNA binding, the value of free energy of interaction, the position and schemes of potential binding sites (BS) were calculated using the MirTarget program. To identify miRNAs whose targets are genes of the TF MYB family, a search of 738 miRNAs BS in mRNAs of 124 MYB family genes of $O$. sativa was performed. 56 genes were identified as targets for 40 miRNAs. The BS of osa-miR529a-3p, osa-miR414-5p, osa-miR2919, osa-miR28685p, osa-miR2097-5p, osa-miR156a-5p, osa-miR1442-5p in the mRNAs of the O. sativa MYB family genes were located in 5'UTR, while osa-miR818a-3p BS in the mRNA of MYB family genes of the O. sativa were located in 3'UTR. The osa-miR5075-3p and osa-miR159c-3p BS were located in 5'UTR and CDS mRNA of the O. sativa MYB family genes. osa-miR2102 binds to mRNAs of 21 MYB genes. The free energy of interaction of osa-miR2102-5p with the mRNA of these genes varied. osa-miR5809-3p had BS in the mRNA of nine target genes. osa-miR5075-3p and osa-miR5833-5p had four and six target genes, respectively. The remaining miRNAs had only one or two target genes. Studying the binding of 125 miRNAs to the mRNAs of 258 genes of the T. aestivum MYB family resulted in a finding that 48 genes were targets for 28 miRNAs. miRNA BS in the mRNA of MYB family genes were located only in the CDS. taemiR159b-3p, tae-miR164-5p and tae-miR444b-3p had BS in the mRNA of seven genes. tae-miR10518-5p had five target genes. For tae-miR5084-3p, tae-miR171a-3p, and taemiR10517-5p, targets were mRNA of three genes. tae-miR9676-5p, tae-miR9666a-3p, tae-miR9662b-3p, tae-miR5384-3p, tae-miR398-3p, tae-miR319-3p, tae-miR1127b-3p had two BS in the mRNA MYB genes. The remaining miRNAs had only one target gene. The target genes from MYB family of $O$. sativa and T. aestivum for miRNAs have been identified, the associations of which were identical for both plant species. 